\title{
Lactate dehydrogenase and aspartate transaminase of the cerebrospinal fluid in patients with brain tumours, congenital hydrocephalus, and brain abscess
}

\author{
S. R. DHARKER, R. S. DHARKER, AND B. D. CHAURASIA \\ From the Departments of Neurosurgery and Anatomy, G. R. Medical College and \\ J.A. Group of Hospitals, Gwalior, M.P., India
}

SYNOPSIS The diagnostic value of CSF lactate dehydrogenase and aspartate transaminase in cases of brain tumours (except for CSF AST in the benign tumours), congenital hydrocephalus, and brain abscess is established. Tumour cyst fluids show a higher enzymatic activity than does the CSF. The two enzyme estimations do not help in differentiating the supratentorial from the infratentorial tumours. CSF AST is superior to CSF LD in discriminating the malignant and benign tumours, in so far as the AST is increased selectively in malignancy. Estimates of CSF LD are slightly superior to those of CSF AST, both in incidence of abnormality and the degree of their rise.

Reports on lactate dehydrogenase (LD) and aspartate transaminase (AST) (formerly termed glutamic oxalacetic transaminase) activities of the cerebrospinal fluid (CSF) in patients with brain tumours are not uniform, and study of these enzymes in the CSF in congenital hydrocephalus and brain abscess has not been done. Fleisher et al. (1957) found normal CSF LD and AST activities in 20 patients with CNS tumours, including four metastatic carcinomas. The normal CSF LD (Jakoby and Jakoby, 1958) and CSF AST (Green et al., 1957; Mann et al., 1960) in various types of brain tumours were also noted subsequently. However, Green et al. $(1958,1959)$ recorded a rise of both enzymes in the CSF in benign as well as malignant tumours. Still others emphasised the increased activity of one (Wróblewski et al., 1958; Mellick and Bassett, 1964) or both (Davies-Jones, 1969) enzymes in the malignant tumours alone, which were either primary or secondary in origin. The LD activity of tumour cyst fluid has also been recorded as raised in malignant tumours (Szliwowski and Cumings, 1961), which is said to be more marked in secondary carcinomas (Buckell et al., 1970).

Address for correspondence: Dr S. R. Dharker, 6 Near Power House, Lashkar, Gwalior, M.P., 474001, India.

(Accepted 18 June 1976.)

\section{METHODS}

The CSF LD and AST activities were estimated in 30 cases of brain tumours, 10 cases of congenital hydrocephalus, and two cases of brain abscess. The enzymes were also estimated in the cyst fluid of eight tumours of the series. The brain tumours included five astrocytomas, four medulloblastomas, four ependymomas, four tuberculomas, three brain stem gliomas, two glioblastomas, two haemangioblastomas, two tumours of the third ventricle, a craniopharyngioma, a meningioma, a cerebellopontine angle epidermoid, and a posterior fossa midline tumour. Of these, 15 were supratentorial and another 15 infratentorial. Out of 30 tumours 25 were proved by histology, and the other five (brain stem gliomas 3, tumour of the posterior third ventricle 1 , posterior fossa midline tumour 1) were named and categorised according to their gross features, clinical behaviours, and investigations. Thus, apart from the tumour of the posterior third ventricle and the posterior fossa midline tumour, 20 tumours were malignant and eight benign. A parallel study on 25 neurologically normal subjects formed the basis of the normal control values for comparison.

The CSF was collected during ventriculography or pneumoencephalography in cases of the brain tumours, during iophendylate ventriculography through the widened coronal suture in cases of congenital hydrocephalus, and during incidental lumbar punc- 
ture in the control cases. The LD and AST activities of the CSF were estimated by King's colorimetric methods $(1960,1965)$, which are still preferred over the spectrophotometric procedures when a group of tests needs to be done simultaneously; it saves time and, moreover, in the latter method an ultra-violet spectrophotometer is needed unless an instrument which will read at $365 \mathrm{~nm}$ is available, and temperature control may not be easy (Varley, 1969). In Varley's experience the colorimetric technique which he used correlates with the spectrophotometric at least as well as some other published variants he had tried. King's unit of LD is defined as that activity which would oxidise $1.0 \mu \mathrm{mol}$ lactate in one hour at $37^{\circ} \mathrm{C}$ and is expressed per $100 \mathrm{ml} \mathrm{CSF}$; to convert it to international units per litre, or $\mathrm{m}-\mathrm{I}$. U/ml, it should be multiplied by two-thirds (Varley, 1969). Similarly, the unit of AST is the activity in $100 \mathrm{ml} \mathrm{CSF}$ which will transform $1 \mu \mathrm{mol}$ amino-acid to $1 \mu \mathrm{mol}$ ketoacid in one hour at $37^{\circ} \mathrm{C}$ under the specified conditions (King, 1960). Daly and Jordan (1959), after reviewing various units proposed for expressing the transaminase activity, suggested that King's unit be used for colorimetric determinations. The recorded data were analysed statistically; the mean, standard deviation, and confidence limits were calculated and the comparisons were made by Student's $t$ test.

\section{RESULTS}

The mean LD content of the control group was 92.5 units $/ 100 \mathrm{ml}$ with a standard deviation of \pm 21.0 and the confidence limits of 81.3 to 100.7 units. The mean AST value was 15.5 units $/ 100 \mathrm{ml}$ with a standard deviation of \pm 4.4 and the confidence limits of 13.6 to 17.4 units.

The LD activity was higher than the upper normal limits (100.7 units) in $27(90 \%)$ cases of brain tumours, and in all the cases of hydrocephalus and brain abscess. The AST was also raised in similar percentages of patients of the three groups, though in lesser proportions than the LD. The rise of both enzymes was uniformly higher in the tumour cyst fluid than in the CSF.

Table 1 shows statistical calculations of the LD and AST values in the various groups investigated, including the controls, whereas comparison of the groups with the controls, using Student's $t$ test, is shown in Table 2. The results are significant at the $5 \%$ probability level in all the groups, except for the CSF AST values in benign tumours $(t=1.778,0.1<\mathrm{P}\rangle$ 0.05 ) which are insignificant. This establishes statistically the diagnostic value of CSF LD and AST in brain tumours (except for CSF AST in benign tumours), congenital hydrocephalus and brain abscess.

Table 3 compares different groups with one another, and shows the relative superiority of one enzyme over the other in the diagnosis of these groups. These enzymes are significantly higher in the brain cyst fluid than in the CSF of such tumour cases, in malignant tumours than in the benign, and in brain abscess than in the brain tumours. However, the difference between the supratentorial and infraten-

TABLE 1

STATISTICAL CALCULATIONS

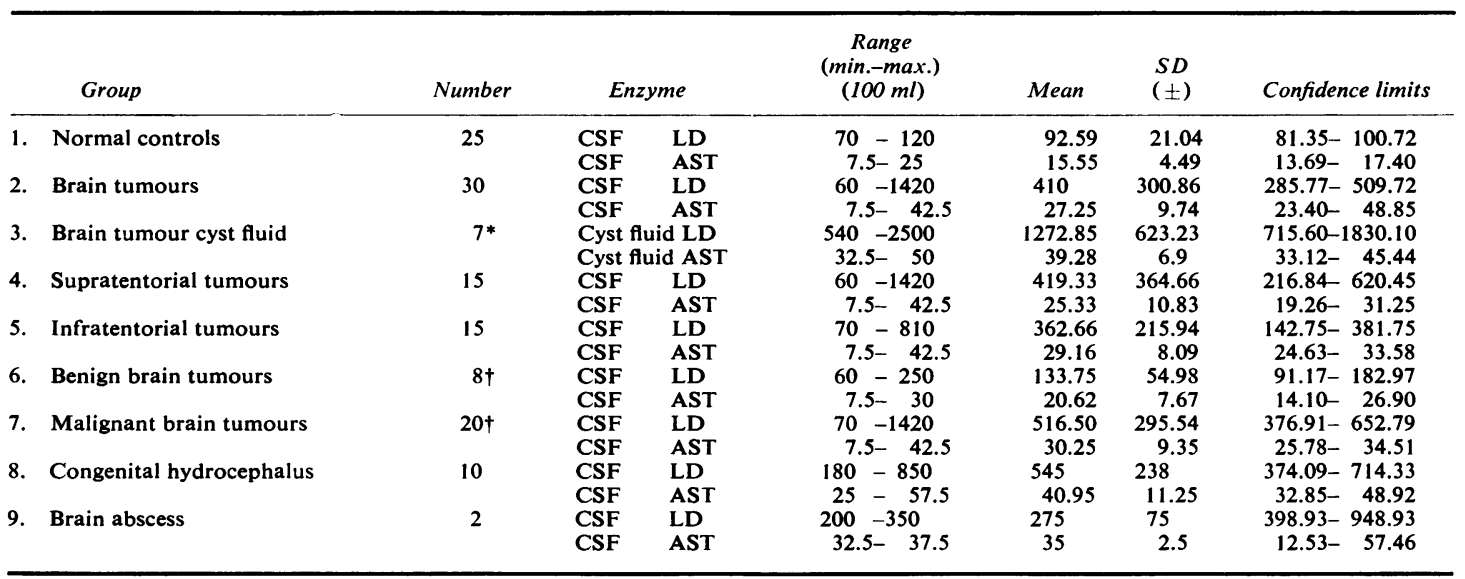

*Cyst fluid LD value of one case and cyst fluid AST value of another case were not considered for statistical reasons.

$\dagger$ Histological nature of one tumour of the posterior third ventricle and one posterior fossa midline tumour could not be ascertained. 
TABLE 2

COMPARISON OF GROUPS WITH NORMAL CONTROLS

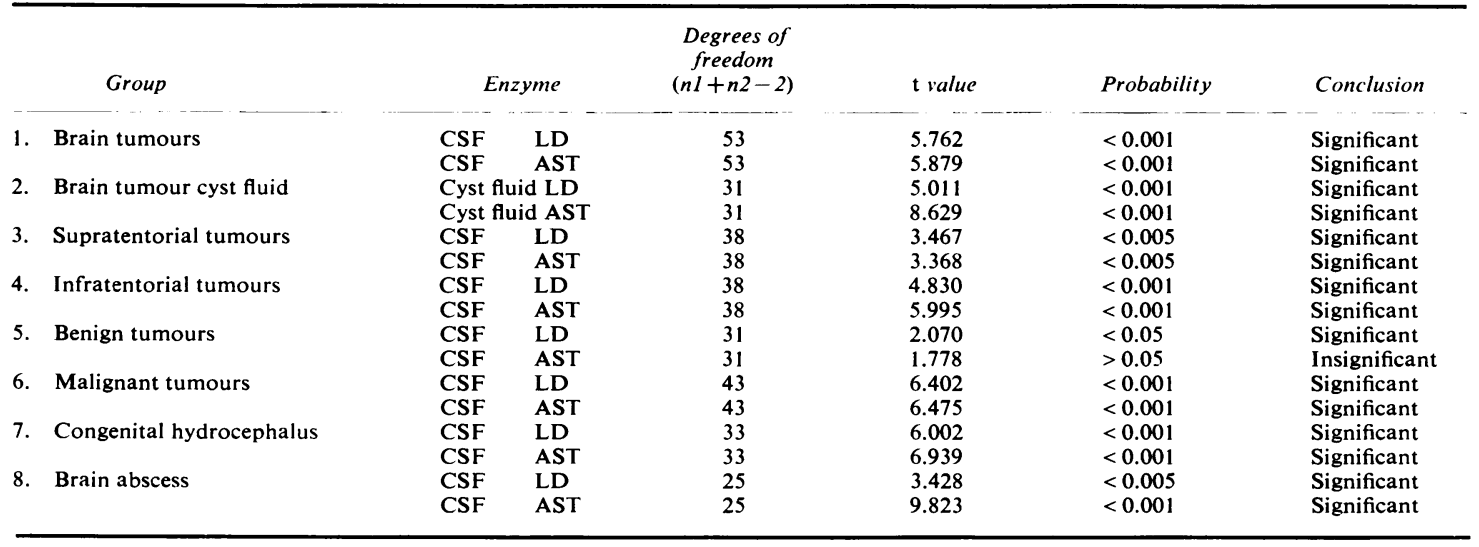

torial tumours is insignificant, and likewise the raised values of CSF LD in congenital hydrocephalus and brain tumours do not show any statistical difference. The difference between the CSF LD and CSF AST values in benign tumours is apparent in Table 2 , where LD values are significantly raised above the normal figures and the CSF AST is unaltered. On the other hand, CSF AST, instead of CSF LD, estimations are helpful in differentiating congenital hydrocephalus from the brain tumours (Table 3 ).

\section{DISCUSSION}

Normal LD and AST activities of CSF in patients with primary brain tumours were reported by Fleisher et al. (1957), Green et al. (1957), Katzman et al. (1957), Wróblewski et al. (1957), Mann et al. (1960) and Davies-Jones (1969). However, the present study revealed raised values of CSF LD and AST in 90 percent of similar patients; those having normal CSF enzymes had mostly supratentorial tuberculomas and in one case a brain stem glioma. These results are in partial agreement with those of Green et al. (1958) and Mellick and Bassett (1964), because their observed frequencies of raised enzymatic activities were lower than the present ones.

The CSF LD and AST activities in both supratentorial and infratentorial tumours are significantly higher than those in the control group (Table 2), and the difference between the two groups of tumours is not significant (Table 3 ). This would refute the claims that the extent of increased intracranial pressure could be related to the increased enzymatic activity of the CSF. Further analysis of these tumours, with

TABLE 3

COMPARISON OF GROUPS WITH ONE ANOTHER

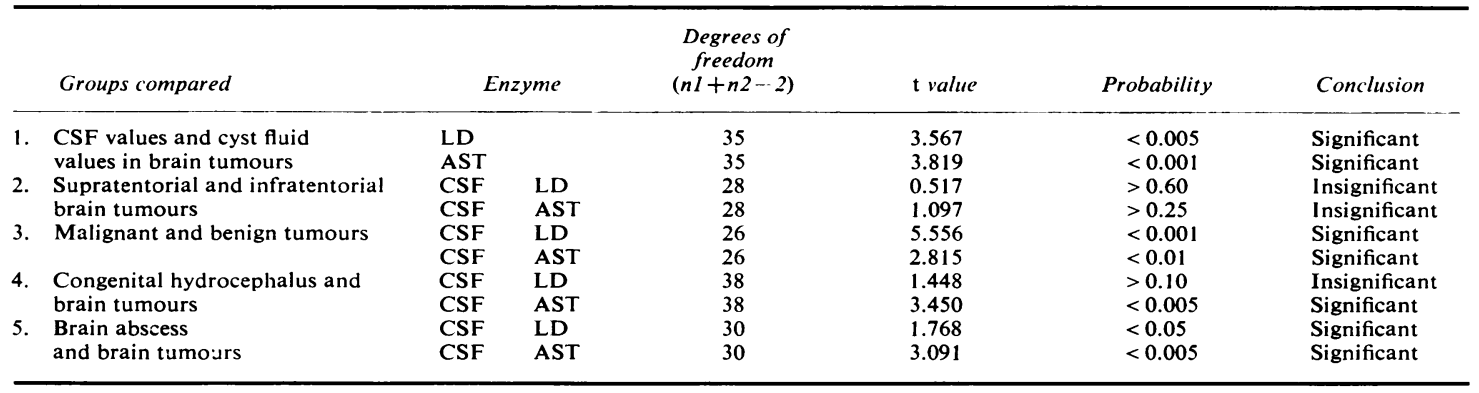


regard to their benign or malignant nature, shows that CSF LD in both types is uniformly higher than the normal values, whereas CSF AST is raised in malignant tumours only. Comparison of the malignant and benign tumours with each other revealed a statistically significant difference. These findings suggest a diagnostic superiority of CSF AST over the CSF LD in differentiating the malignant from the benign tumours, since the transaminase activity is increased selectively and significantly with malignancy, although lactate dehydrogenase also shows a significant difference in its rise in two types of the tumours. The correlation between the increased enzyme activity of the CSF and the degree of malignancy of a tumour was suggested by Green et al. (1959), Mellick and Bassett (1964) and Davies-Jones (1969). This is partly supported by the present findings, because the highest records of the enzyme activities in the malignant tumours is not uniform. Viewing the histological types of the tumours, it appears that tuberculomas, whether supratentorial or infratentorial, are associated with either normal or a little over the normal CSF LD and AST activities. The other tumours showed varying degrees of rise of the two enzymes in the CSF which was more pronounced with LD than with AST. Thus, in general, the estimations of CSF LD are slightly superior to those of CSF AST, both in incidence of abnormality and in degree of its rise.

The lactate dehydrogenase activity of the tumour cyst fluid is regarded as a better index of the degree of malignancy than its activity in the CSF (Green et al., 1958; Szliwowski and Cumings, 1961; Buckell and Robertson, 1965; Buckell et al., 1970). This is supported by the present observations whereby dehydrogenase estimations in the cyst fluid of three benign tumours (colloid cyst of third ventricle, craniopharyngioma, and the epidermoid) were uniformly lower than those in the malignant tumours. However, this does not hold good for the transaminase activity, which shows overlapping figures in the two categories of benign and malignant tumours. The cyst fluid shows consistently higher enzymatic activities than the CSF.

The CSF LD and AST activities in patients with congenital hydrocephalus were significantly higher than in the normal subjects. It is interesting to note (Table 3) that behaviour of the CSF LD in congenital hydrocephalus and brain tumours is more or less similar without any statistical difference $(t=1.448$, $0.2<\mathrm{P}>0.1)$, whereas CSF AST is significantly higher in hydrocephalus than in the brain tumours.

The CSF enzymes were significantly raised above the normal values in both cases of brain abscess of this series. The raised figures differ significantly from those in the tumour cases (Table 3). Although two cases of brain abscess do not form a representative group, these preliminary observations should prompt further studies on larger series to ascertain their validity.

\section{REFERENCES}

Buckell, M., and Robertson, M. C. (1965). Enzyme studies in cerebral tumours. Lactate dehydrogenase, glucose phosphate isomerase and alkaline phosphatase in plasma, ventricular cerebrospinal fluid and tumour cyst fluid from cases of glioma and secondary carcinoma. British Journal of Cancer, 19, 83-91.

Buckell, M., Crompton, M. R., Robertson, M. C., and Barnes, G. K. (1970). Lactate dehydrogenase in cerebral cyst fluids. Total activity and isoenzyme distribution as an index of malignancy. Journal of Neurosurgery, 32, 545-552.

Daly, J., and Jordan, A. (1959). Serum-transaminase levels. Lancet, 1, 256-257.

Davies-Jones, G. A. B. (1969). Lactate dehydrogenase and glumatic oxalacetic transaminase of the cerebrospinat fluid in tumours of the central nervous system. Journal of Neurology, Neurosurgery, and Psychiatry, 32, 324-327.

Fleisher, G. A., Wakim, K. G., and Goldstein, N. P.ๆ (1957). Glutamic oxalacetic transaminase and lactic de- $\infty$ hydrogenase in serum and cerebrospinal fluid of patients with neurologic disorders. Mayo Clinic Proceedings, 32, 188-197.

Green, J. B., Oldewurtel, H. A., Forster, F. M., and Sanchez-Longo, L. P. (1957). Cerebrospinal fluid 8 glutamic oxalacetic transaminase activity in neurologio diseases. Neurology (Minneap.), 7, 313-322.

Green, J. B., Oldewurtel, H. A., and Forster, F. M. (1959) Glutamic oxalacetic transaminase (GOT) and lactic dehydrogenase (LDH) activities. Study in the cerebrospinal fluid of brain tumor patients, in normal human brain and in brain tumor homogenates. Neurology (Minneap.), 9, 540-544.

Green, J. B., Oldewurtel, H. A., O'Doherty, D. S., and Forster, F. M. (1958). Cerebrospinal fluid transaminase and lactic dehydrogenase activities in neurologic diseases. Archives of Neurology and Psychiatry (Chic.), 80, 148-156.

Jakoby, R. K., and Jakoby, W. B. (1958). Lactic dehydrogenase of cerebrospinal fluid in the differential diagnosis of cerebrovascular disease and brain tumor. Journal of Neurosurgery, 15, 45-51.

Katzman, R., Fishman, R. A., and Goldensohn, E. S. (1957). Glutamic oxalacetic transaminase activity in spinal fluid. Neurology (Minneap.), 7, 853-855.

King, J. (1960). A study of human serum transaminases. Journal of Medical Laboratory Technology, 17, 1-21.

King, J. (1965). Practical Clinical Enzymology. van Nostrand: London.

Mann, S. H., DePasquale, N., and Paterson, R. (1960). Cerebrospinal fluid glutamic oxalacetic acid transaminase in patients receiving electroconvulsive therapy 
and in neurologic diseases. Neurology (Minneap.), 10, 381-390.

Mellick, R. S., and Bassett, R. L. (1964). The cerebrospinal fluid glutamic oxalacetic transaminase activity in neurological diseases. Lancet, 1, 904-906.

Szliwowski, H. B., and Cumings, J. N. (1961). The diagnostic value of the chemical examinations of cerebral cyst fluids. Brain, 84, 204-211.
Varley, H. (1969). Practical Clinical Biochemistry, 4th edn, pp. 279, 294. Heinemann: London.

Wróblewski, F., Decker, B., and Wróblewski, R. (1957). Activity of lactic dehydrogenase in spinal fluid. American Journal of Clinical Pathology, 28, 269-271.

Wróblewski, F., Decker, B., and Wróblewski, R. (1958). The clinical implications of spinal fluid lactic dehydrogenase activity. New England Journal of Medicine, 258, 635-639. 